\title{
Optimizing particle size distribution measured by laser diffraction technique for estimating soil hydraulic properties
}

\author{
Ahmed Yehia Mady ${ }^{1,2,3}$ and Evgeny Shein ${ }^{1,2}$ \\ ${ }^{1}$ Faculty of Soil Science, Soil Physics and Reclamation Department, Lomonosov Moscow State University, Leninskie \\ Gory, 119991, Moscow, Russia. \\ ${ }^{2}$ Soil Science Institute named after V.V. Dokuchaev, Pyzhevsky per.7, 119017, Moscow, Russia. \\ ${ }^{3}$ Faculty of Agriculture, Soil Science Department, Ain Shams University, \\ Hadayek Shoubra, 11241, Cairo, Egypt. \\ [Received: August 29, 2019 Accepted: October 23, 2019]
}

\section{Abstract}

Particle size distribution (PSD) is the fundamental characteristic that gives information about soil physical properties. It is the essential predictor used in most predictive programs for predicting water flux, solute, and heat transport in soil. Predictive programs such as Rosetta, RETC, and HYDRUS-1D are usually used PSD measured by the pipette method (PM), whereas the usage of PSD measured by laser diffraction technique (LDT) yields a large estimation error in heavy soil. The aim of the work was to optimize PSD measured by LDT for approaching to PSD measured by PM by suggesting Pedotransfer functions (PTFs). Furthermore, it is to evaluate the efficiency of PSD calculated by the proposed PTFs for estimating soil hydraulic properties using Rosetta program. Particle size distribution was measured by two methods: LDT and PM using the same dispersion factor (sodium pyrophosphate solution 4\%). Proposed PTFs were derived for calculation of particle size distribution using a linear regression between PSD measured by LDT as an independent variable and PSD measured by PM as a dependent variable. PSD calculated using proposed PTFs leads to optimize values of PSD measured by LDT for approaching to values of PSD measured by PM. Optimizing PSD by proposed PTFs was suitable for calculating soil hydraulic parameters using Rosetta program with a little estimation error, for agro-soddy podzolic with soil texture as silty loam and silty clay loam.

Keywords: Pipette method, predictive programs, pedotransfer functions, optimizing laser diffraction method, silty loam

\section{Introduction}

Particle size distribution (PSD) is an important and fundamental parameter used to recognize different soil characteristics. It plays a big role in providing information about soil physical properties such as soil bulk density, information entropy (IE) (Martín et al., 2017), pore size distribution, water holding capacity, soil carbon storage, and erodibility (Van Looy et al., 2017; Minasny and McBratney, 2018). Furthermore, pollutant movement, land degradation, and soil quality are affected by particle size distribution (Kusuma et al., 2018; Chidozie et al., 2019). PSD is usually used as a predictor variable in pedotransfer functions (PTFs) and predictive programs based on sedimentation method measurements (Schaap et al., 2001; Šimůnek et al., 2008; Jorda et al., 2015; Sedaghat et al., 2016). PSD measured by PM is widely used for estimation of soil hydro-physical properties such as saturated hydraulic conductivity (Shein et al., 2015; Mady and Shein, 2018a), soil water retention curve (Makó et al., 2014; Lamorski et al., 2014) and hysteresis of soil water retention curve (Lamorski et al., 2017; Shein and Mady, 2018; Mady and Shein, 2018b; Shein et al., 2019). Also, it has been used for calculation of soil thermal-physical properties such as thermal diffusivity (Mady and Shein, 2018c). There are many programs used PSD as an input data such as Rosetta, RETC, and HYDRUS-1D programs (Šimůnek et al., 2008). However, the accuracy of output data is related to the methods of PSD measurement. There are various methodologies used for measurement of PSD such as sieving method, sedimentation method (SM), electro-resistance particle counting, optical estimation using image analysis, and laser diffraction method (Blott and Pye, 2012; Dobrowolski et al., 2012). Sedimentation methods have mainly relied on Stoke's law such as hydrometer and pipette methods. They are accurate, but they are consuming time and money. The efficiency of sedimentation method is dependent on removing flocculating agents such as organic matter, calcium carbonate, salts, and iron oxides. Moreover, it relies on

\footnotetext{
*Email: ahmed_mady@agr.asu.edu.eg
} 
concentration of dispersing agent and suspension temperature. Also, the clay fraction $<0.002 \mathrm{~mm}$ needs a long time for its estimation reaching to eight hours. Recently, laser diffraction technique has been performed for PSD measurement. Although LDT is a simple and fast method for PSD estimation, however it has estimated a smaller content of clay and larger content of silt compared to sedimentation method measurements (Di Stefano et al., 2010; Polakowski et al., 2014; Mady and Shein, 2017). The efficiency of LDT is based mainly on a dispersing agent (Ryżak and Bieganowski, 2011; Makó et al., 2014; Mady and Shein, 2017). Moreover, the shape of sand particles influences the PSD estimation using LDT (Polakowski et al., 2014). The usage of PSD measured by LDT could yield larger estimation error when used in predictive programs for estimation of hydro-physical properties. PTFs is commonly utilized to translate data easy to measure into data difficult to measure (Bouma, 1989). So, the aim of this study was to optimize PSD values measured by LDT for approaching to PSD values measured using PM by suggesting PTFs equations. Moreover, PSD calculated by proposed PTFs was used for calculation of soil hydraulic properties using Rosetta program in order to evaluate the efficiency of PSD calculated by the proposed PTFs.

\section{Material and Methods}

\section{Definition of soil sampling}

Thirty soil samples were collected from agro-soddy podzolic soil (Albic Glossic Retisols), Pushkin oblast, Moscow, Russia. Soil samples were randomly selected according to the differences in the depth. Soil texture was classified as silty loam and silty clay loam soils. Soil samples were split into two classes; i) the first class was twenty soil samples used as a training dataset for suggesting PTFs and developing PSD measured by LDT; ii) the second class was ten soil samples used as a testing dataset for estimating the efficiency of PSD calculated using proposed PTFs.

\section{Particle size distribution (PSD)}

PSD was determined using two methodologies:

\section{Sedimentation method (SM)}

The pipette method (PM) is one of the main sedimentation methods used for PSD measurement. PSD was measured using the pipette method (PSD-PM) based on Stoke's law according to Gee and Bauder (1986) and Gee and Or (2002). Soil samples were prepared by sieving soil samples at a diameter of sieve $2 \mathrm{~mm}$, and then sodium pyrophosphate solution $4 \%$ concentration was added. PSD was classified according to USDA classification as sand fraction $(2-0.05) \mathrm{mm}$, silt fraction $(0.05-0.002) \mathrm{mm}$ and clay fraction $<0.002 \mathrm{~mm}$.

\section{Laser diffraction technique (LDT)}

PSD was measured using laser diffraction technique (PSD-LDT) by comfort Analysette-22 with a wavelength $623 \mathrm{~nm}$ according to (Eshel et al., 2004) based on Mie theory. Helium-neon laser was used as a light source. Soil samples were prepared by sieving soil samples at a diameter $0.25 \mathrm{~mm}$, then sodium pyrophosphate solution $4 \%$ concentration was added as a dispersing factor.

The laser diffraction technique has relied on the principle that the particles which have small size are given a large certain angle of the refracted light beam, and vice versa the particles which have large size are given a small certain angle. Also, the intensity of the refracted beam is referring to the quantity of the particles at any refraction angle.

\section{Determination of PTFs for optimizing PSD measured by LDT}

PTFs was proposed using linear regression for twenty soil samples used as training dataset in order to increase the efficiency of PSD measured using LDT, by recalculating PSD using proposed PTFs (PSD-PTFs) as the following:

Sand content was calculated using PTFs by a linear regression between sand measured by LDT as an independent variable $(\mathrm{X})$ and sand measured by $\mathrm{PM}$ as a dependent variable $(\mathrm{Y})$ for twenty soil samples, $\mathrm{R}^{2}=0.69$ as Figure 1.

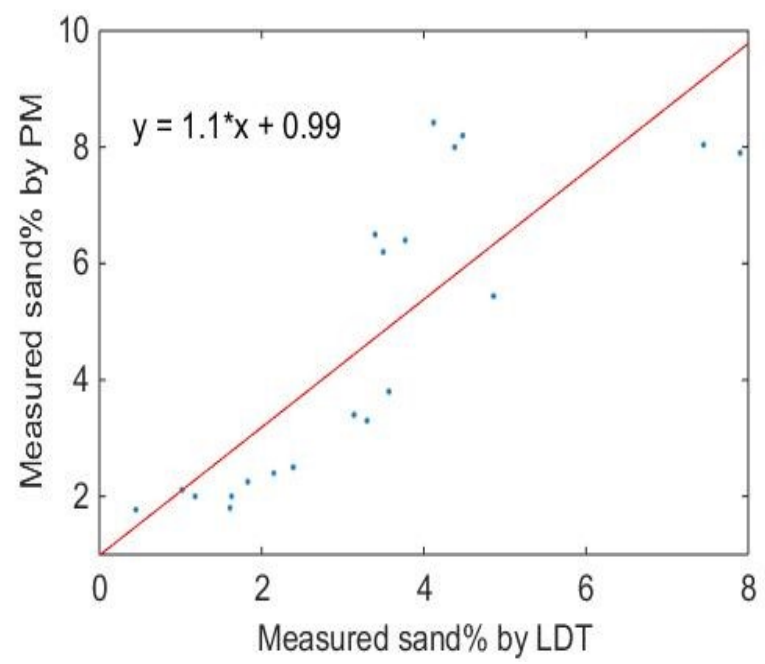

Figure1: Linear regression between sand measured by LDT and PM

Sand ${ }_{\text {PTFs }}=1+1.1$ Sand $_{\text {LDT }}$ 
Moreover, Clay content was calculated using PTFs by a linear regression between clay measured by LDT as an independent variable (X) and clay measured by PM as a dependent variable $(\mathrm{Y})$ for twenty soil samples, $\mathrm{R}^{2}=0.79$ as Figure 2.

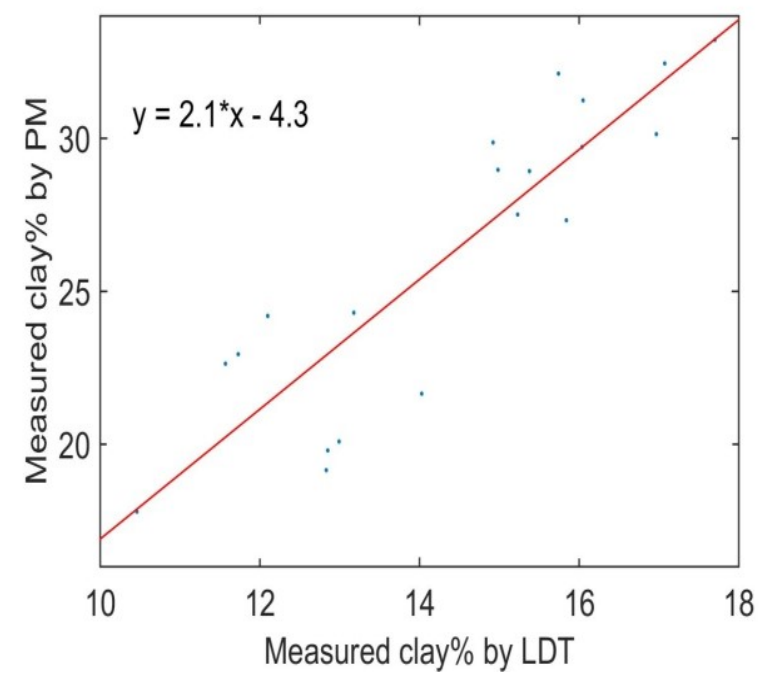

Figure 2: Linear regression between clay measured by LDT and PM

Clay PTFs $=-4.3+2.12$ Clay ${ }_{\text {LDT }}$

There are not any correlation or determination coefficients between silt measured by LDT and the measured by PM, R ${ }^{2}$ was 0.01 . So, silt content was calculated as the following equation;

$$
\text { Silt PTFs } \%=100-(\text { Sand PTFs } \%+\text { Clay PTFs } \%)
$$

where, sand PTFs, silt PTFs, and clay PTFs are referring to the percentage of PSD- PTFs.

\section{Estimation of soil hydraulic properties}

Rosetta and RETC programs are widely used for estimation of the parameters of van Genuchten (1980), saturated hydraulic conductivity (Schaap et al., 2001), and unsaturated hydraulic conductivity (Mualem 1976). Rosetta program is utilized as one of the following categories: (soil texture); (particle size distribution (PSD)); (PSD and bulk density); (PSD, bulk density and water content at field capacity); and (PSD, bulk density, water content at field capacity and wilting point).

$$
\begin{aligned}
& \left.\frac{\theta-\theta_{r}}{\theta_{s}-\theta_{T}}=1+[\alpha h]_{n}\right]^{-m} \\
& \mathrm{~m}=1-\frac{1}{n} \quad \text { Se }=\frac{\theta-\theta_{\mathrm{r}}}{\theta_{s}-\theta_{\mathrm{r}}},
\end{aligned}
$$

where, $\theta_{\mathrm{r}}$ and $\theta_{\mathrm{s}}$ are referring to the residual and saturated water contents, respectively; $\alpha$ is the inverse of bubbling pressure; $\mathrm{n}$ is a pore-size distribution index. Se is an effective saturation degree.

In this study, in order to determine the accuracy of PSD calculated using proposed PTFs, the parameters of model's van Genuchten (1980) " $\theta \mathrm{r}, \theta \mathrm{s}, \alpha$, and n" were calculated using Rosetta program based on the PSD measured by three methods; PSD-PM, PSD-LDT, and PSD-PTFs.

\section{Statistical analysis}

The efficiency of PSD measured by LDT and PSD calculated using the proposed PTFs was calculated using Root Mean Square Error (RMSE) (Tietje and Hennings, 1996).

$$
\text { RMSE }=\sqrt{\frac{\sum_{i=1}^{n}\left(y_{i}-\hat{y_{i}}\right)^{2}}{N}}
$$

where, $y_{i}$ is refer to the measured value, $\hat{y}_{i}$ refers to the calculated value, and $\mathrm{N}$ is the number of testing dataset (ten soil samples).

\section{Software programs}

Software programs were used in this study including the Rosetta program database, RETC, and MATLAB programs.

\section{Results and Discussion}

\section{Evaluation of PSD measured by LDT and PM}

Statistical analysis of the testing dataset of ten soil samples is shown in Figure 3. The mean value of sand percentage measured by PM was larger than that measured by LDT as Figure 3a. Also, the mean value of the percentage of clay measured by PM was larger than its measured by LDT Figure $3 \mathrm{c}$. While the mean value of silt percentage measured by PM was smaller than that measured by LDT as Figure 3b. Furthermore, the percentage of sand measured by PM was 2.5 times larger than its measurement by LDT. Also, the percentage of clay measured by PM was 1.9 times larger than its measurement by LDT. While the percentage of silt measured by PM was 2.1 times smaller than its measurement by LDT (Di) (Stefano et al., 2010; Polakowski et al., 2014). The reason for the difference between the sedimentation method and laser diffraction technique is due to the non-spherical shape of soil particles (Fedotov et al., 2007). The sedimentation method assumes that the particles have a spherical shape and having the same density based on Stoke's law, but it is true rarely. Also, the difference is commonly attributed to the optical properties 
which change with shape, diameter, and refractive angle of the particles based on the Mie theory (Ryżak and Bieganowski, 2011; Sochan et al., 2012; Polakowski et al., 2014; Makó et al., 2014) for laser diffraction technique. Moreover, there is no agreement between PSD- PM and PSD- LDT as Figure 3. So, utilizing PSD-LDT in the predictive programs tend to large estimation error especially, if this difference leads to a change in soil texture (Mady and Shein, 2017). sand and clay calculated by PTFs, respectively, Figure 3a and Figure 3c. Whereas, the mean value of the percentage of silt measured by LDT decreased from $82.82 \%$ to $71.83 \%$ for silt calculated by PTFs as Figure 3b. Box plot in Figure 4 shows that the difference between PSD-PM and PSD-PTFs was smaller than the difference between PSD-PM and PSDLDT as the percentages of sand, silt, and clay. Also, the mean error of the difference between PSD-PM and PSDPTFs was close to zero than those differences between PSD-
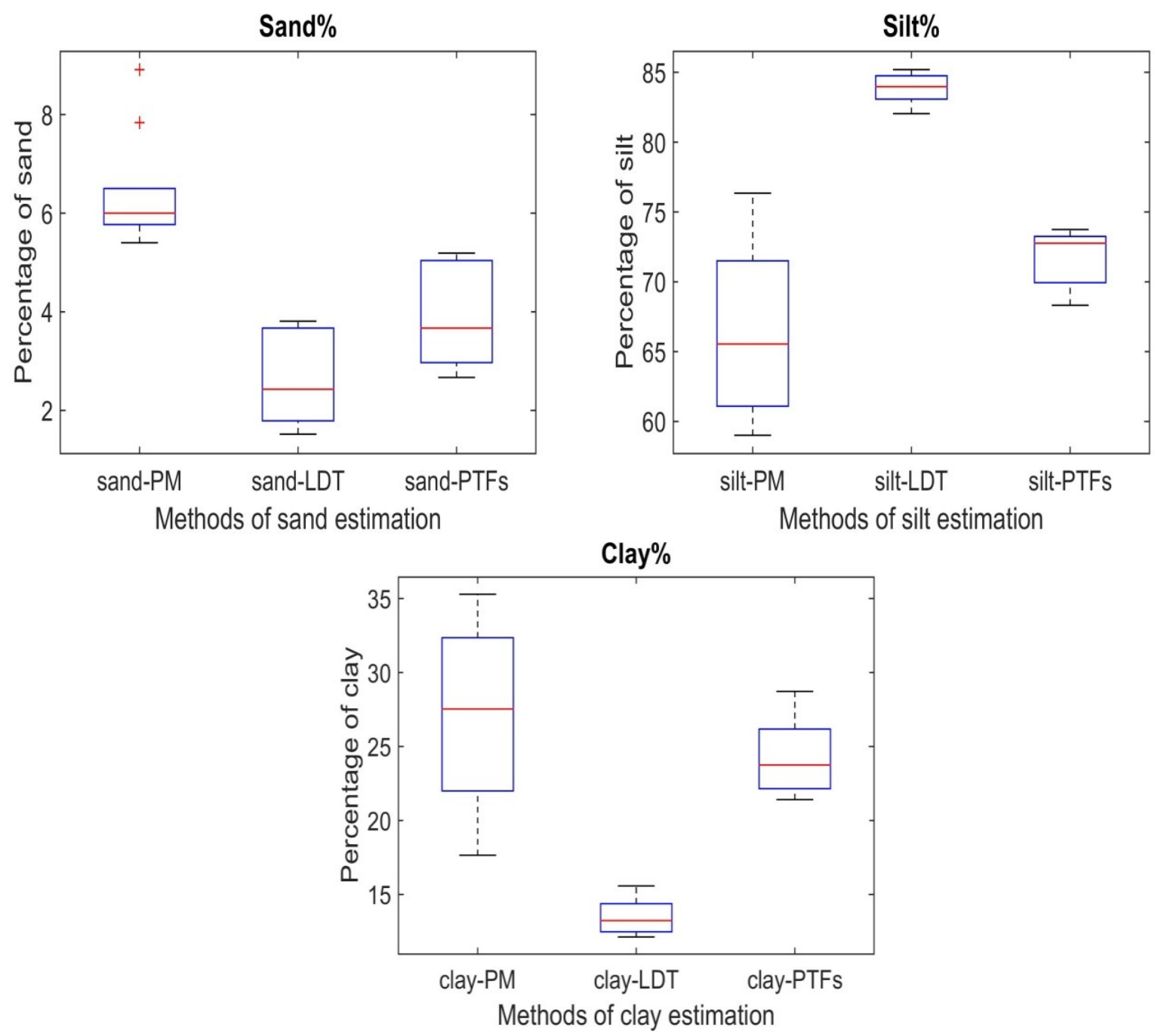

Figure 3: The statistics analysis of testing dataset for PSD measured by PM, LDT and calculated by PTFs

\section{Optimizing PSD measured by LDT using proposed PTFs}

Statistical analysis of PSD- PTFs is shown in Figure 3 $(a, b, c)$ for the testing dataset. The mean values of the percentages of sand and clay measured by LDT were increased from $2.64 \%$ and $13.47 \%$ to $3.9 \%$ and $24.26 \%$ for
PM and PSD-LDT as Figure $4(a, b, c)$. Furthermore, the relative error was decreased for the percentages of sand, silt, and clay which was measured by LDT from $3.81 \%, 17.11 \%$, and $13.36 \%$ to $2.55 \%, 5.12 \%$, and $0.43 \%$ for those calculated using PTFs, respectively. Moreover, RMSE was decreased for PSD- LDT as the percentages of sand, silt, and clay from $4.1 \%, 18.14 \%$, and $14.32 \%$ to $1.33 \%, 2.47 \%$ 
and $2.87 \%$ for those calculated using PTFs (PSD-PTFs), respectively. Also, RMSE decreased 3 times for calculated sand, 7.34 times for calculated silt, and 4.98 times for calculated clay using proposed PTFs (PSD-PTFs). The reason of that is the correlation coefficient between PSDPM and PSD- PTFs was larger than between PSD- PM and PSD- LDT, especially the percentage of silt. The correlation coefficient between measured silt by PM and its measurement by LDT was 0.309 . Whereas, it was 0.66 between silt measured by PM and the calculated using PTFs. Proposed PTFS lead to optimize PSD measured by LDT, and PSD calculated by PTFs was approached to PSD measured by PM as Figure $3(a, b, c)$ and Figure $4(a, b, c)$.

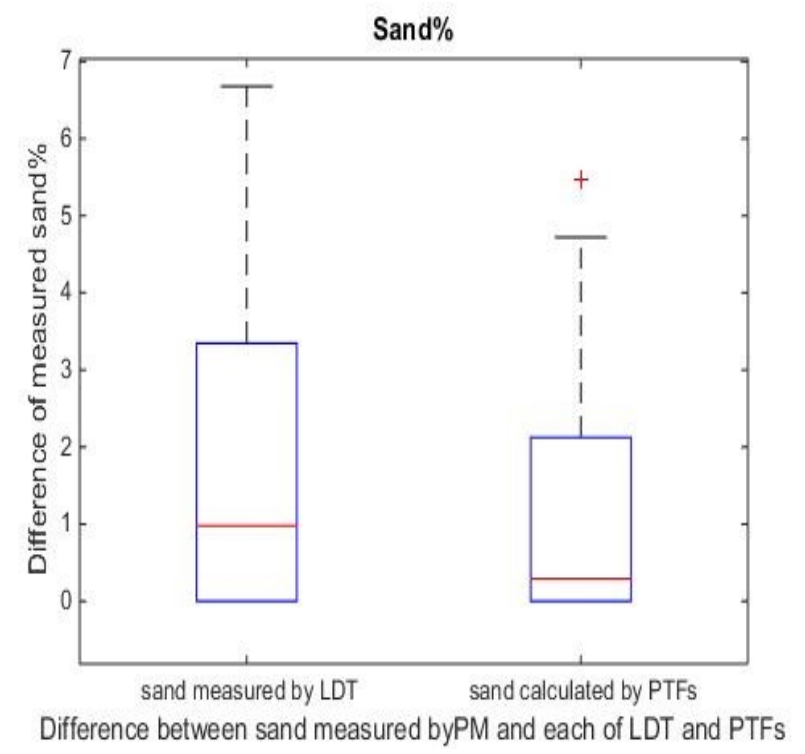

\section{Soil hydraulic properties}

Particle size distribution is commonly used as a predictor variable in PTFs equations and predictive programs for estimation of soil water retention curve (Schaap et al., 2001; Lamorski et al., 2014). It has a great effect on soil hydraulic properties involving the parameters of van Genuchten $\theta \mathrm{r}, \theta \mathrm{s}, \alpha$, and $\mathrm{n}$. In order to determine the efficiency of PSD calculated by PTFs, soil hydraulic properties involving the parameters of van Genuchten were calculated using Rosetta program based on PSD-PM, PSDLDT and PSD-PTFs. Statistical analysis for the testing dataset shows that the mean values of the parameters of van Genuchten $(\theta \mathrm{r}, \theta \mathrm{s}, \alpha$, and $\mathrm{n})$ calculated by PSD-PM were as
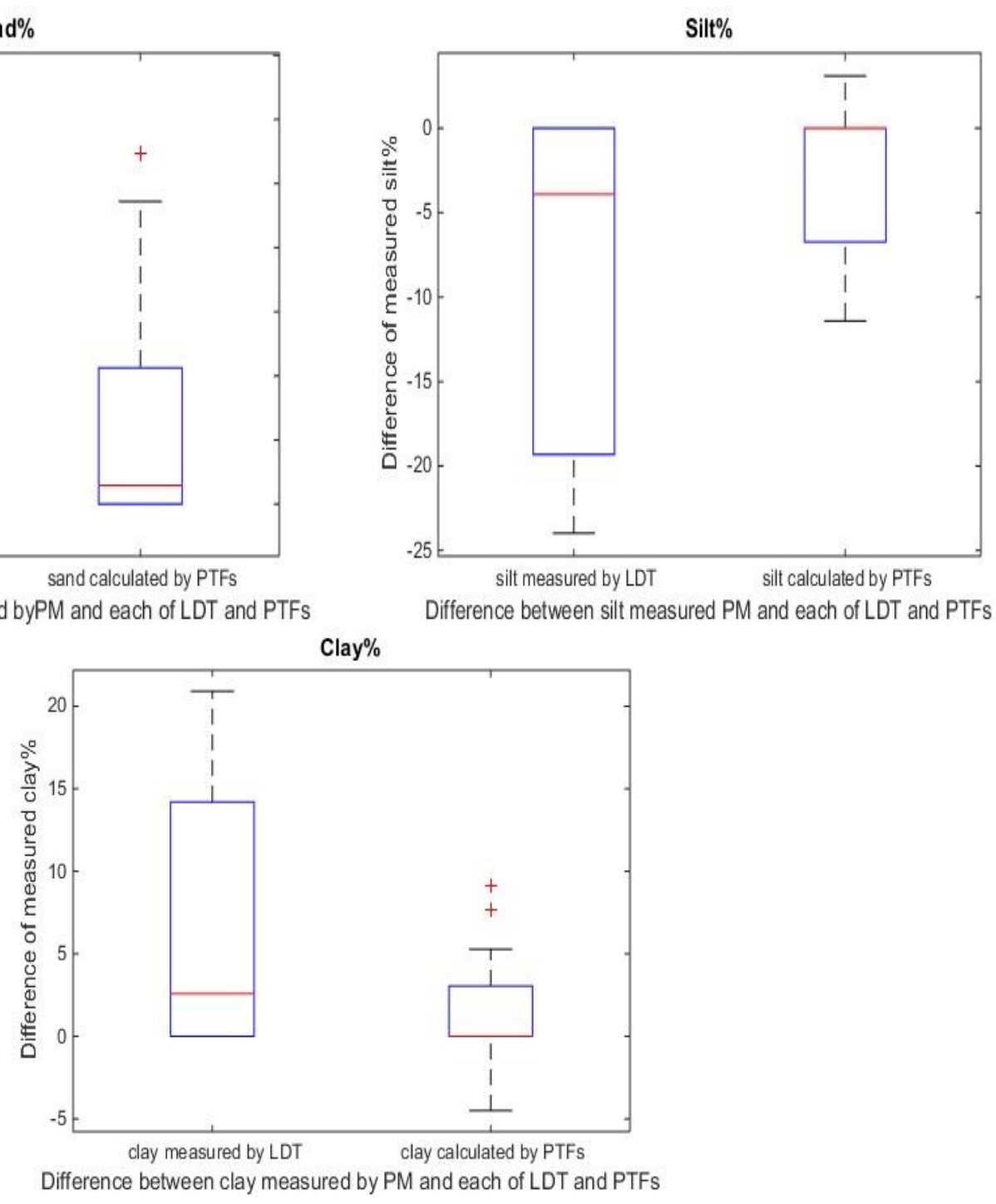

Figure 4: The difference between PSD measured by PM and each of LDT and PTFs 
$0.0838 \mathrm{~cm}^{3} . \mathrm{cm}^{-3}, 0.468 \mathrm{~cm}^{3} \cdot \mathrm{cm}^{-3}, 0.0072 \mathrm{~cm}^{-1}$ and 1.55, whereas those values were $0.0682 \mathrm{~cm}^{3} \cdot \mathrm{cm}^{-3}, 0.479 \mathrm{~cm}^{3} \cdot \mathrm{cm}^{-3}$, $0.0070 \mathrm{~cm}^{-1}$, and 1.62 for the parameters calculated by PSDLDT, respectively. The parameters of van Genuchten $(\theta \mathrm{r}$ and $\alpha$ ) calculated based on PSD- LDT were usually smaller than the calculated by PSD- PM. The reason of that is related to the percentage of clay measured by LDT was smaller than its measurement by PM as Figure 3c (Di Stefano et al., 2010; Mady and Shein, 2017). While the calculated parameters ( $\theta \mathrm{s}$ and $\mathrm{n}$ ) based on PSD- LDT were larger than those calculated by PSD- PM. The reason of that is due to the percentage of sand measured using LDT was smaller than its measured using PM as Figure 3a. Utilizing of PSD- PTFs that gives rise to decreasing RMSE for the parameters of van Genuchten ( $\theta \mathrm{r}, \theta \mathrm{s}, \alpha$, and $\mathrm{n}$ ) calculated by PSD- LDT from $\left(0.016 \mathrm{~cm}^{3} . \mathrm{cm}^{-3}, 0.015 \mathrm{~cm}^{3} . \mathrm{cm}^{-3}, 0.0011\right.$ $\mathrm{cm}^{-1}$, and 0.083$)$ to $\left(0.004 \mathrm{~cm}^{3} . \mathrm{cm}^{-3}, 0.007 \mathrm{~cm}^{3} . \mathrm{cm}^{-3}, 0.0093\right.$ $\mathrm{cm}^{-1}$, and 0.039 ) for those parameters calculated using PSD-
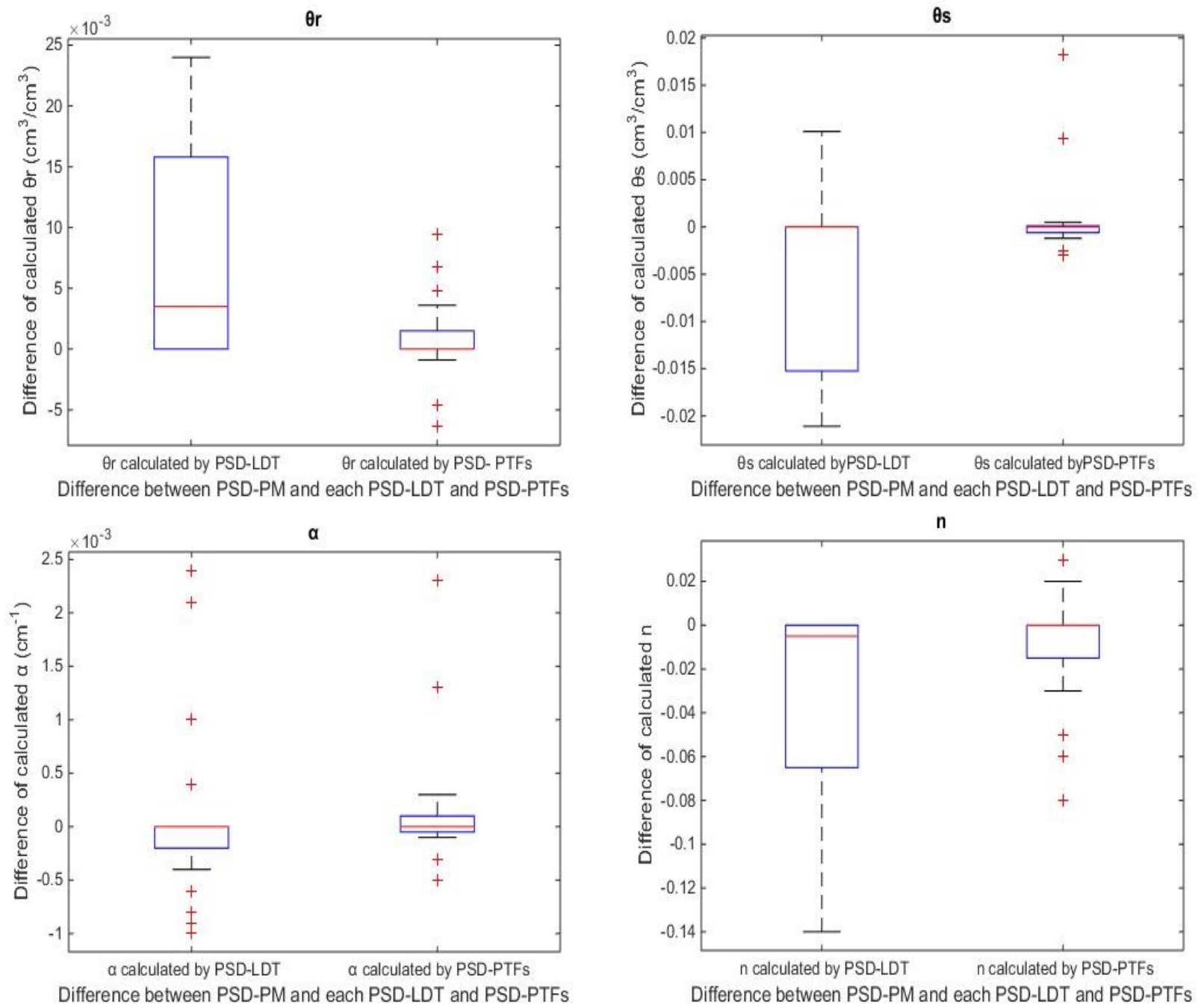

Figure 5: The difference between the parameters of van Genuchten measured based on PSD-PM and each of PSDLDT and PSD- PTFs

Table 1: RMSE for the parameters of van Genuchten calculated using PSD measured by LDT and proposed PTFs

\begin{tabular}{|c|c|c|c|c|c|c|c|}
\hline \multirow[t]{2}{*}{$\begin{array}{l}\text { Statistical } \\
\text { analysis }\end{array}$} & \multicolumn{3}{|c|}{$\begin{array}{l}\text { The calculated parameters of van Genuchten } \\
\text { using PSD- LDT }\end{array}$} & \multicolumn{4}{|c|}{$\begin{array}{l}\text { The calculated parameters of van Genuchten } \\
\text { using PSD- PTFs }\end{array}$} \\
\hline & $\theta \mathrm{r} \mathrm{cm}^{3} \cdot \mathrm{cm}^{-3} \quad \theta \mathrm{s} \mathrm{cm}^{3} \cdot \mathrm{cm}^{-3}$ & $\alpha \mathrm{cm}^{-1}$ & $\mathrm{n}$ & $\theta \mathrm{r} \mathrm{cm}^{3} \cdot \mathrm{cm}^{-3}$ & $\theta \mathrm{s} \mathrm{cm}^{3} \cdot \mathrm{cm}^{-3}$ & $\alpha \mathrm{cm}^{-1}$ & $\mathrm{n}$ \\
\hline RMSE & 0.016 & 0.0011 & 0.083 & 0.004 & 0.007 & 0.0096 & 0.039 \\
\hline
\end{tabular}


PTFs as Table 1. The reason of that is attributed to the mean values of the parameters of van Genuchten $(\theta \mathrm{r}, \theta \mathrm{s}, \alpha$, and $\mathrm{n}$ ) calculated by PSD- PTFs was close to those calculated by PSD- PM. They were $\left(0.0822 \mathrm{~cm}^{3} . \mathrm{cm}^{-3}, 0.466 \mathrm{~cm}^{3} \cdot \mathrm{cm}^{-3}\right.$, $0.007 \mathrm{~cm}^{-1}$ and 1.57) for $\theta \mathrm{r}, \theta \mathrm{s}, \alpha$, and $\mathrm{n}$ calculated by (PSDPTFs), respectively. In addition to statistical analysis, the box plot in Figure $5(\mathrm{a}, \mathrm{b}, \mathrm{c}, \mathrm{d})$ shows that the difference between the parameters of van Genuchten values $(\theta \mathrm{r}, \theta \mathrm{s}, \alpha$, and n) calculated by PSD-PM and PSD- PTFs was smaller than the differences between the values of the parameters calculated by PSD-PM and PSD-LDT. Also, the mean error of the difference between the parameters of van Genuchten values calculated by PSD-PM and PSD- PTFs was close to zero and it did not have an experimental error as Figure 5 $(\mathrm{a}, \mathrm{b}, \mathrm{c}, \mathrm{d})$. But those differences between the parameters calculated by PSD-PM and PSD- LDT have had large experimental error especially ( $\theta \mathrm{r}$ and $\mathrm{n}$ ) as (Figure $5 \mathrm{a}$, and Figure 5d).

\section{Conclusion}

Laser diffraction technique is a simple and fast technique used for measurement of particle size distribution. However, LDT is given a larger estimation error comparing with sedimentation methods in heavy soil and when used it as a predictor variable in predictive programs. There is the correlation coefficient between both sand and clay contents measured by PM and those measured by LDT. But there is no correlation between silt measured by PM and its measurement by LDT. PSD values measured by LDT were optimized by suggesting PTFs with linear regression equations in order to approach to values of PSD measured by PM. The calculated PSD using PTFs was close to PSD measured by PM. The calculated PSD using PTFs tends to decrease the experimental error and the values of RMSE for the parameters of van Genuchten $\theta \mathrm{r}, \theta \mathrm{s}, \alpha$, and $\mathrm{n}$ calculated using Rosetta program based on particles size distribution. The usage of calculated PSD using PTFs leads to optimize measured PSD by LDT and enables it to use in Rosetta program for calculating soil hydraulic properties with little estimation error, for agro-soddy podzolic soil with soil texture silty loam and silty clay loam.

\section{References}

Bouma, J. 1989. Using soil survey data for quantitative land evaluation. Advances in Soil Science 9: 177-213.

Blott, S.J. and K. Pye. 2012. Particle size scales and classification of sediment types based on particle size distributions: Review and recommended procedures. Sedimentology 59: 2071-2096. doi: 10.1111/j.13653091.2012.01335.x

Chidozie, E.I., I.F. Ifeanyi, O.M. Johnbosco, I.A. Onyekachi, C.C. Anthony and O.M. Obinna N.U. Gift,
D. George, A.S. Emeka, T. Raza and M.O. Glory. 2019. Assessment of hydraulic conductivity and soil quality of similar lithology under contrasting land use and land cover in humid tropical Nigeria. Soil \& Environment 38(1): 75-80. DOI:10.25252/SE/19/61419

Di Stefano, C., V. Farro and S. Mirabile. 2010. Comparison between grain-size analyses using laser diffraction and sedimentation methods. Biosystems Engineering 106(2): 205-2015.

Dobrowolski, R., A. Bieganowski, P. Mroczek and M. Ryzak. 2012. Role of periglacial processes in epikarst morphogenesis: A case study from Chelm Chalk Quarry, Lublin upland Eastern Poland. Permafrost Periglac. Process 23: 251-266.

Eshel, G., G.J. Levy, U. Mingelgrin and M.J. Singer. 2004. Critical evaluation of the use of laser diffraction for particle-size distribution analysis. Soil Science Society of America Journal 68(3): 736-743.

Fedotov, G.N., E.V., Shein, V.I. Putlynev, T.A., Arkhangel'skaya, A.V. Eliseev and E.Y. Milanovskii. 2007. Physicochemical bases of differences between the sedimentometric and laser diffraction techniques of soil particle-size analysis. Eurasian Soil Science 40(3): 281-288.

Gee, G.W. and J.W. Bauder. 1986. Particle-size analysis. p.383-411. In: Methods of Soil Analysis. J.H. Klute (ed.). Soil Science Society of America Book Series No.9, Agron. Monogr. Madison.

Gee, G.N. and D. Or. 2002. Particle size analysis. p.225293. In: Methods of Soil Analysis. D.I. Dan and G.C Topps. (eds.). Soil Science Society of America Book Series No.5, Madison.

Jorda, H., M. Bechtold, N. Jarvis and J. Koestel. 2015. Using boosted regression trees to explore key factors controlling saturated and near-saturated hydraulic conductivity. European Journal of Soil Science 66: 744-756. DOI: https://doi.org/10.1111/ejss.12249

Kusuma, M.N., W. Hadi and B. Wirjodirdjo. 2018. Preliminary study of infiltration gallery for water treatment towards Universal Access 2019 in Indonesia. Soil \& Environment 37(1): 83-88. DOI:10.25252/SE/18/51284

Lamorski, K., A. Bieganowski, M. Ryżak, A. Sochan, C. Sławiński and W. Stelmach. 2014. Assessment of the usefulness of particle size distribution measured by laser diffraction for soil water retention modeling. Journal of Plant Nutrition \& Soil Science 177: 803-813.

Lamorski, K., J. Šimůne, C. Sławiński and J. Lamorska. 2017. An estimation of the main wetting branch of the soil water retention curve based on its main drying branch using the machine learning method. Water 
Resources Research 53: 1539-1552. DOI: 10.1002/2016WR019533.

Mady, A.Y. and E.V. Shein. 2017. Comparison between particle size distribution as a predictor of pedotransfer functions using laser diffraction and sedimentation methods. International Journal of Soil Science 12(2): 65-71. DOI: http://dx.doi.org/10.3923/ijss.2017.65.7

Mady, A.Y. and E.V. Shein. 2018a. Support vector machine and nonlinear regression methods for estimating saturated hydraulic conductivity. Moscow University Soil Science Bulletin 73 (3): 129-133.

Mady, A.Y. and E. Shein. 2018b. Modelling and validation hysteresis in soil water retention curve using tomography of pore structure. International Journal of Water 12 (4): 370-381.

Mady, A.Y. and E. Shein. 2018c. Estimating soil thermal diffusivity using Pedotransfer functions with nonlinear regression. The Open Agriculture Journal 12: 164-173.

Makó, A., K. Rajkai, H. Hernádi and G. Hauk. 2014. Comparison of different settings and pre-treatments in soil particle-size distribution measurement by laserdiffraction method. Agrokémia és Talajtan 63(1): 1928.

Martín, M. A., M. Reyes and F.J. Taguas. 2017. Estimating soil bulk density with information metrics of soil texture. Geoderma 287: 66-70, https://doi.org/10.1016/j.geoderma.2016.09.008.

Minasny, B. and A.B. McBratney. 2018. Limited effect of organic matter on soil available water capacity. European Journal of Soil Science 69: 39-47.

Mualem Y. 1976. A new model for predicting the hydraulic conductivity of unsaturated porous media. Water Resources Research 12 (3): 513-522.

Polakowski, C., A. Sochan, A. Bieganowski, M. Ryzak, R. Földényi and J. Tóth. 2014. Influence of the sand particle shape on particle size distribution measured by laser diffraction method. International Agrophysics 28(2): 195-200.

Ryżak, M. and A. Bieganowski. 2011. Methodological aspects of determining soil particle-size distribution using the laser-diffraction method. Journal of Plant Nutrition \& Soil Science 174: 624-633.

Schaap, M.G., F.J. Leij and M.Th. van Genuchten. 2001. Rosetta: A computer program for estimating soil hydraulic parameters with hierarchical pedotransfer functions. Journal of Hydrolog 251(3-4): 163-176.
Sedaghat, A., H. Bayat, and A.A. Safari Sinegani. 2016. Estimation of soil saturated hydraulic conductivity by artificial neural networks ensemble in smectitic soils. Eurasian Soil Science 49(3): 347-357.

Shein, E.V. and A.Y. Mady. 2018. Hysteresis of the water retention curve: wetting branch simulation based on the drying curve. Moscow University Soil Science Bulletin 73(3): 124-128.

Shein, E.V., A.Y. Mady and El.A. Mohamed. 2015. Soil saturated hydraulic conductivity assessment by direct and pedotransfer functions methods. Biogeosystem Technique 6(4): 396-400.

Shein, E.V., A.Y. Mady and L.I. Il'in. 2019. Validation of HYDRUS-1D for predicting of soil moisture content with hysteresis effect. Biogeosystem Technique 6(1):5964.

Sochan, A., A. Bieganowski, M. Ryżak, R. Dobrowolski and P. Bartmin'ski. 2012. Comparison of soil texture determined by two dispersion units of master sizer 2000. International Agrophysics 26(1): 99-102. doi: 10.2478/v10247-012-0015-9

Šimůnek, J., M.T. van Genuchten, and M. Šejna. 2008. Development and applications of the HYDRUS and STANMOD software packages and related codes. Vadose Zone Journal 7: 587-600.

Tietje, O. and V. Hennings. 1996. Accuracy of the saturated hydraulic conductivity prediction by pedo-transfer functions compared to the variability within FAO textural classes. Geoderma 69(1-2): 71- 84 .

van Genuchten, M.T. 1980. A closed-form equation for predicting the hydraulic conductivity of unsaturated soils. Soil Science Society of America Journal 44(5): 892-898.

Van Looy, K., J. Bouma, M. Herbst, J. Koestel, B. Minasny and U. Mishra U. Mishra, C. Montzka, A. Nemes, Y.A. Pachepsky, J. Padarian, M.G. Schaap, B. Tóth, A. Verhoef, J. Vanderborght, M.J. van der Ploeg, L. Weihermüller, S. Zacharias, Y. Zhang and $\mathrm{H}$. Vereecken. 2017. Pedotransfer functions in earth system science: challenges and perspectives. Reviews of Geophysics 55: 1199-1256. 